\title{
AN ADAPTIVE APPROACH FOR SEGMENTATION OF 3D LASER POINT CLOUD
}

\author{
Z. Lari, A. F. Habib, E. Kwak \\ Department of Geomatics Engineering, University of Calgary, Calgary, Alberta, Canada T2N 1N4 -
}

(zlari, ahabib, ekwak@ucalgary.ca)

\section{Commission V, WG V/3}

KEY WORDS: Segmentation, Kd-tree data structure, Adaptive cylinder neighbourhood, Clustering attributes, Octree space partitioning

\begin{abstract}
:
Automatic processing and object extraction from 3D laser point cloud is one of the major research topics in the field of photogrammetry. Segmentation is an essential step in the processing of laser point cloud, and the quality of extracted objects from laser data is highly dependent on the validity of the segmentation results. This paper presents a new approach for reliable and efficient segmentation of planar patches from a 3D laser point cloud. In this method, the neighbourhood of each point is firstly established using an adaptive cylinder while considering the local point density and surface trend. This neighbourhood definition has a major effect on the computational accuracy of the segmentation attributes. In order to efficiently cluster planar surfaces and prevent introducing ambiguities, the coordinates of the origin's projection on each point's best fitted plane are used as the clustering attributes. Then, an octree space partitioning method is utilized to detect and extract peaks from the attribute space. Each detected peak represents a specific cluster of points which are located on a distinct planar surface in the object space. Experimental results show the potential and feasibility of applying this method for segmentation of both airborne and terrestrial laser data.
\end{abstract}

\section{INTRODUCTION}

Laser scanning is considered as a leading technology for the acquisition of high density three-dimensional spatial information. Due to the variety of $3 \mathrm{D}$ objects and massive amount of points, extraction of valuable spatial information from 3D laser scanning data is difficult and time consuming. Segmentation is the fundamental step in information extraction from 3D laser point clouds. The objective of the segmentation process is to cluster points with similar attributes into homogeneous regions and introduce some level of organization to the data before the extraction of useful information. Many methodologies have been suggested for the segmentation of $3 \mathrm{D}$ laser data in the past decade, which are generally categorized in three classes: region growing, model fitting methods, and clustering of attributes.

The region growing method proposed by Besl and Jain (1988) includes two steps: identification of the seed points and growing them based on predefined criteria such as proximity of points and planarity of surfaces. Several research efforts proposed improvements to the functionality of the region growing segmentation. Tovari and Pfeifer (2005) introduced a region growing segmentation method for airborne laser data. They used the estimated normal vector for each point and its distance to the growing plane as the similarity criteria for growing the seed point. Rabbani et al. (2006) utilized the surface roughness to group points belonging to the same surface. $\mathrm{Pu}$ and Vosselman (2006) proposed a region growing method for terrestrial laser data based on proximity of points and global planarity criteria. These methods are highly dependent on selected seed points and errors in defining these points will affect the segmentation process (Besl and Jain, 1998).

The model fitting methods, which are developed based on fitting geometric primitive shapes, can be applied for mathematical representation of planar surfaces. The points which are confirmed by the mathematical representation are grouped as one segment. A well-known algorithm based on the model fitting approach is the RANdom SAmple Consensus (RANSAC) proposed by Fischer and Bolles, (1981). This algorithm was applied for automatic processing of point cloud with the aim of 3D building modeling (Tarsh-Kurdi et al., 2007). The main shortcoming of model fitting approaches is their inefficiency and spurious segmentation results when dealing with different point cloud sources (Filin, 2002).

The segmentation method based on clustering of attributes is a robust approach for the identification of homogenous patterns in the data. This method mainly comprises two processes: attribute computation, and clustering the data based on the computed attributes. Since this method is highly dependent on the quality of derived attributes, they should be computed precisely to produce the best separation among different classes. These techniques generate a voting scheme in the attribute space which is constructed using an accumulator array. The dimension of this accumulator array is dependent on the number of the utilized attributes for clustering. Vosselman and Dijkman (2001) used the principal of the Hough transform (Hough, 1962) for segmentation of planar surfaces in a 3D laser point cloud. In this method, each laser point defines a plane in the 3D attribute space. So, the laser points on the same planar surface will intersect at the position in the attribute space that corresponds to the slopes and distance of the planar surface. Filin and Pfeifer (2006) introduced a segmentation method based on the normal vectors derived using a slope adaptive neighbourhood. They used the slopes of the normal vector in the $\mathrm{X}$ and $\mathrm{Y}$ directions and height difference between the point and its neighbourhood as the clustering attributes. This height difference attribute was also used to guarantee the distinction between parallel planes, which share the same normal vector slopes. Biosca and Lerma (2008) suggested a fuzzy clustering approach in combination with a similarity-based cluster merging for segmentation of a terrestrial laser point cloud. Kim et al. (2007) proposed a method for segmentation of planar 
patches in 3D laser data. They used the magnitudes of surface normals derived from two defined origins as the clustering attributes. The two origins should be located in a way that minimizes the risk of ambiguity. The segmentation methods based on clustering attributes are efficient and flexible for the identification of homogeneous regions in the data. The major drawbacks of these methods are lack of computational efficiency when dealing with multidimensional attributes for a massive amount of points and their dependency on the neighbourhood definition and the local point density.

Extraction of man-made structures is of great importance in 3D laser data processing. These structures usually comprise multiple planar surfaces which should be segmented within data before feature extraction. So, this paper will outline an alternative approach for the segmentation of planar patches based on cluster analysis in attribute space. Figure 1 shows the main stages of the proposed methodology.

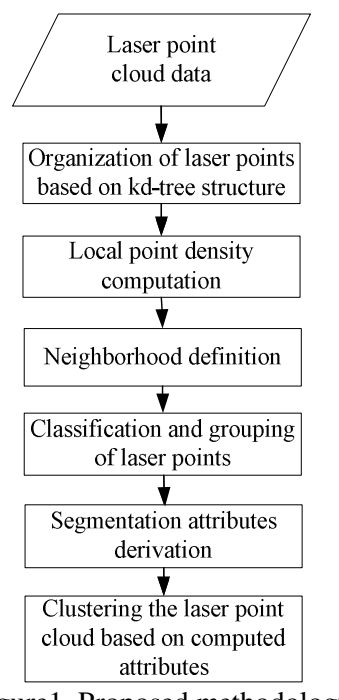

Figure1. Proposed methodology

This paper is organized as follows: Section 2 describes the organization of $3 \mathrm{D}$ laser data using kd-tree structure, the process of neighbourhood definition based on the local point density and physical shape of surfaces, and classification and grouping of laser data based on defined neighbourhood system. Section 3 presents the suggested approach for segmentation of 3D laser point cloud which is essentially based on clustering the computed attributes. Section 4 provides the experimental results of the proposed method. Finally, section 5 summarizes the main achievements reached in this work.

\section{ADAPTIVE NEIGHBOURHOOD DEFINITION AND POINT CLASSIFICATION}

The original laser scanner point cloud does not provide explicit information about the distribution of points; therefore, it is necessary to structure the irregular laser point cloud, define the relationship between points and then search for the nearest neighbouring points before processing them. In this paper, a kdtree data structure is used for the organization of 3D laser data and computation of the local point density. The adaptive cylinder neighbourhood for each point is then established based on the local point density and physical properties of the object surface.

Finally, 3D laser data are classified and grouped into rough and planar surfaces based on the adaptive neighbourhood definition.

\subsection{Structuring the laser point cloud based on kd-tree structure}

The performance of the clustering based segmentation methods highly depends on the computed attributes for individual laser points. These attributes are derived based on the defined neighbourhood for each point. In order to improve the efficiency of the neighbourhood definition process, some level of organization should be introduced for the 3D laser data before further processing.

The kd-tree data structure, which was recommended by Freidman et al. (1977), is a strategy for sorting and organizing a set of points. This structure is constructed by recursive subdivision of the three-dimensional space. The splitting strategy used in this paper subdivides the space along the longest extent of the data in the $\mathrm{X}, \mathrm{Y}$, or $\mathrm{Z}$ direction. The splitting plane will be perpendicular to the chosen extent direction and pass through the point with median coordinate along the selected extent. The recursive splitting procedure in three dimensions proceeds until all the points are structured in the kd-tree. This kind of space-partitioning process makes the points being stored in a balanced tree (Moore, 1991). The main advantage of such a structure is faster and more efficient searching of the nearest neighbours.

\subsection{Neighbourhood definition}

Neighbourhood definition is the primary step of 3D laser data processing. This definition is a rule that determines the neighbours of each point, and as a result has a great impact on the computed attributes for laser data segmentation.

Different neighbourhood definitions are being used for laser scanning data presently. Triangulated irregular network (TIN) is a neighbourhood system which is defined based on the proximity of points in 2D space (projection of the points on XY plane). The major shortcoming of this neighbourhood definition is that points belonging to different physical objects might be included in the same neighbourhood.

The other type of neighbourhood definition is based on proximity of points in 3D space (Euclidian distance between points). This definition, which is called spherical neighbourhood, corresponds to a sphere with a certain radius whose centre coincides with the point in question (Lee and Schenk, 2001). All points within the sphere are considered as neighbouring points. While using the 3D distance among investigated points, this definition doesn't consider the physical shape of objects; therefore, different physical surfaces may be included in the same neighbourhood.

The shortcomings of aforementioned neighbourhood systems mandate the development of an alternative neighborhood definition which takes proximity of points in 3D space, physical shapes of associated surfaces (Filin and Pfeier, 2005) and local point density parameters into account. Most of the existing neighbourhood definitions do not consider the varying point densities in laser datasets within the segmentation process. The main objective of this research is the development a segmentation approach which is suitable for both airborne and terrestrial laser data with varying point densities.

In order to compute the local point density ( $L P D)$ parameter for neighbourhood definition, a bounding sphere is defined which includes $K$ nearest neighbours of the point in question. $K$ is the number of points which are utilized for the local point density computation and assumed to be coplanar. This parameter is determined based on the source of the laser data. These neighbouring points are then sorted based on their distances to the query point. The radius of this bounding sphere, $r_{k}$, is the distance between the point in question and its $K^{\text {th }}$ nearest 
neighbour. So, the local point density (number of points $/ \mathrm{m}^{2}$ ) is calculated using this formula:

$$
L P D=\frac{K}{\pi r_{K}^{2}}
$$

The adaptive cylinder neighbourhood of a point is then determined as follows: At first, a spherical neighbourhood is defined for the point of interest with radius:

$$
r_{S N}=\sqrt{\frac{n}{\pi \times L P D}}
$$

In which, $n$ is the reliable number of the points for definition of a plane while considering the existence of the outliers, and $L P D$ is the local point density.

Afterwards, the best fitted plane for the points in the spherical neighbourhood is computed using an iterative plane fitting process based on Least squares adjustment. The parameters of the best fitted plane are refined in the Least squares adjustment process by assigning the proper weight to the points based on their normal distances to the computed plane. Finally, the adaptive neighbourhood is defined by determination of the points whose normal distances to the best fitted plane are less than half of the cylinder height. The cylinder height is an adaptive parameter which is defined proportional to the expected level of noise $(\sigma)$ in different datasets.

The schematic concept of the adaptive cylinder neighbourhood system is illustrated in Figure 2.

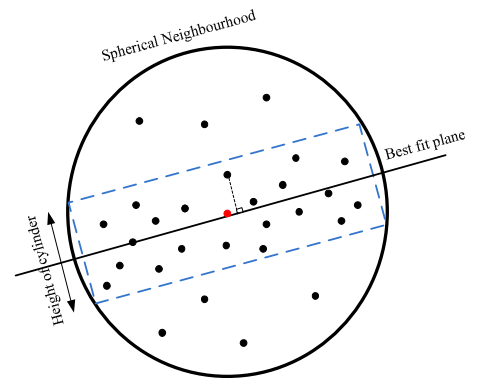

Figure 2. 2D representation of Adaptive cylinder neighbourhood definition

The defined neighbourhood system is implemented based on the local point density, 3D relationships between the points, the local surface trend and the expected level of noise in the data; so, it is suitable for both terrestrial and airborne laser datasets with varying point density and different surface slopes.

\subsection{Classification and grouping of points}

In order to speed up the process of the attributes computation, the dataset should be classified and grouped into rough and planar surfaces. This is carried out based on the defined adaptive cylindrical neighbourhood using a classification measure which is calculated as:

$$
\text { Classification_Measure }=\frac{\text { No. of pnts in the cylindrical neighborhood }}{\text { No. of pnts in the spherical neighborhood }}
$$

If this measure is more than a pre-defined threshold, the point is considered as a part of planar surface; otherwise it is classified as a part of rough surface. Afterwards, a region growing algorithm is employed to group the classified points; in which the seed points are chosen based on the order of points in the established kd-tree. The selected seed points are grown by checking the proximity of neighbouring points in the 3D space. The outcomes of this process are the groups of points which belong to planar surfaces.

\section{SEGMENTATION}

The segmentation is generally defined as the abstraction process of a laser point cloud into distinct subsets of spatially connected points with common attributes. The proposed techniques for segmentation of laser points mainly differ in the methods or criteria which are being used for measuring the similarity among a group of points. In this research, a set of attributes are computed to check the similarity of points in the parameter space. Consequently, the distinct segments can be obtained by clustering the points with similar attributes. The implemented methodology for planar patch segmentation of 3D laser scanning data will be explained in detail in the following section.

\subsection{Attribute computation}

The characteristic attributes for planar patch segmentation are computed based on the adaptive cylindrical neighbourhood defined for each point as shown in section 2.2. To compute the segmentation attributes, the best fitted plane for each point in each planar group should be determined using the iterative plane fitting process. Since, both airborne and terrestrial laser date are dealt with in this research and these datasets comprise planar surfaces with different slopes, a slope-intercept representation of $3 \mathrm{D}$ plane is used firstly to determine the suitable form of the plane for each point. The quality of fitting to slope-intercept representation forms can be used as a measure for selection of best representation. The representation form with the best fitting quality is selected as the most convenient plane for the neighbouring points. The parameters of the best fitted plane are then used for computation of segmentation attributes.

In this research, the coordinates of the origin's normal projection on the best fitted plane derived for each point $\left(X_{0}, Y_{0}\right.$, $\left.Z_{0}\right)$ are utilized as the segmentation attributes. These attributes are computed based on the parameters of the best fitted plane for each point in each group. A 3D accumulator array is then constructed based on the defined attributes for efficient clustering of the planar patches in the attribute space.

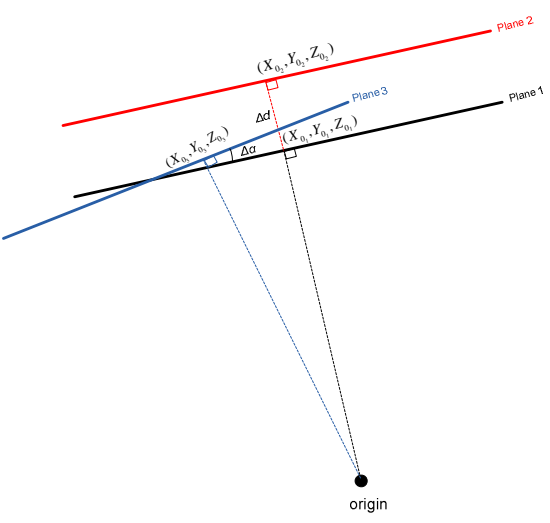

Figure 3. Computed attributes for the segmentation of planes with different slopes and parallel planes

As shown in Figure 3, the major preference of the derived attributes is their uniqueness when clustering planes with different slopes and parallel planes in object space.

\subsection{Clustering of laser point cloud in attribute space}

Once the segmentation attributes are computed and the accumulator array is constructed in the attribute space, a discretization approach should be utilized for the detection and extraction of the accumulated peaks in the attribute space. 
These peaks will represent distinct planar patches in the object space.

Available discretization methods tessellate the attribute space into the cells whose size is determined based on the peaks' extent in attribute space. The main disadvantage of these methods is their large storage and computational requirements when dealing with large accumulator arrays. In this paper, a new method is proposed for finding the peaks in the attribute space. This approach tries to improve the computational efficiency of this process by avoiding unnecessary tessellations of attribute space.

This method, which is developed based on octree space partitioning, is a two-step searching procedure. This algorithm tries to find the approximate peak location firstly (coarse peak searching), and then detect and extract the precise peak location (fine peak searching). In the first step, the attribute space of all of the points in each group is defined and divided into eight equal sub-spaces using an octree space partitioning structure. The splitting process proceeds for sub-spaces including maximum number of attribute points in each stage until the defined sub-space is covered with the sphere whose radius, $r$, is a function of minimum angular separation between detectable planes and minimum distance between distinct parallel planes (Figure 4):

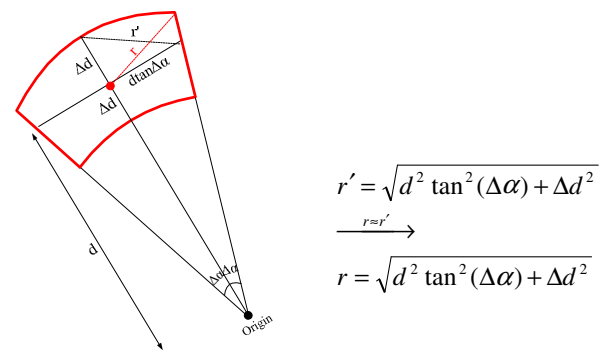

Figure 4. 2D Representation of peak area in attribute space

Based on the derived value for $r$, the coarse peak searching process proceeds until:

$$
\max (\text { sub }- \text { space }- \text { size })<\frac{2 \sqrt{d^{2} \tan ^{2}(\Delta \alpha)+\Delta d^{2}}}{\sqrt{3}}
$$

Where $d$ is the distance of the selected sub-space's center (shown in green color in Figure 5) to the origin, $\Delta \alpha$ is the minimum angular separation between detectable planes and $\Delta d$ is the minimum distance between distinct parallel planes. The selected sub-space which contains the maximum number of the attribute points is considered as the approximate peak location. Figure 5 shows the procedure of coarse peak searching.

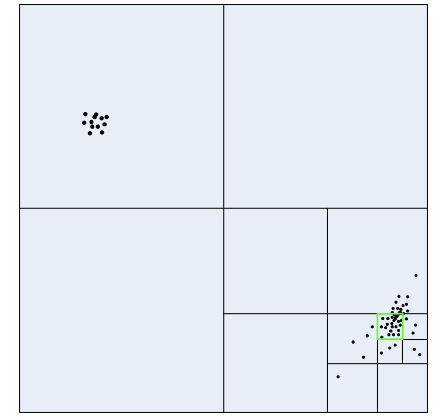

Figure 5. 2D representation of coarse peak searching procedure for finding the approximate peak location

In order to precisely extract a peak, a spherical neighborhood is defined for all the attribute points which are included in the final selected sub-space whose radius equals $r$ (Figure 6). The spherical neighborhood shown in red in Figure 6 is identified as the peak location.

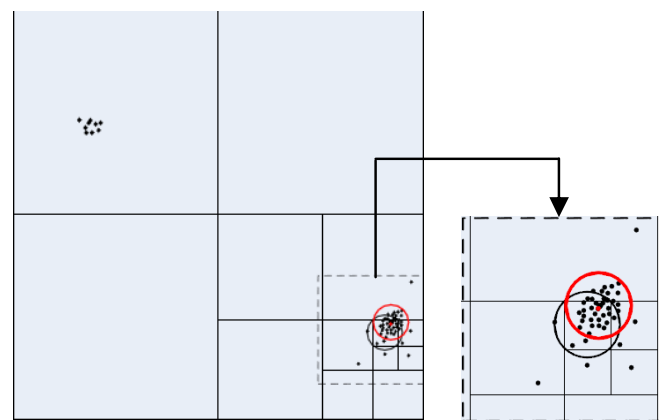

Figure 6. 2D representation of fine peak searching procedure

Once the first peak is detected, the points in the object space whose attributes belong to the peak area are recorded as one cluster and their attributes are removed from the accumulator array. Other peaks are detected using the same procedure until the number of remaining points in the accumulator array is less than a predetermined number of points. This threshold defines the size of minimum detectable cluster. A region growing algorithm is then applied to differentiate the coplanar planes which are disjoint in the object space.

\section{EXPERIMENTAL RESULTS}

The proposed segmentation approach can be applied for both airborne and terrestrial laser data; however, the defined thresholds in this algorithm should be adjusted for different laser datasets based on the scanning system characteristics and segmentation objectives. The test datasets which are selected to evaluate the performance of this method include airborne laser data collected in Switzerland with the Scan2Map mapping system and terrestrial laser data obtained from a complex building façade in University of Calgary campus using a Trimble GS200 3D laser scanner. These datasets include various object features such as walls, flat and gable roofs, terrain surface and vegetation. Table 1 lists the basic characteristics of provided datasets and the thresholds applied for the planar patch segmentation in the proposed method.

\begin{tabular}{|l|c|c|}
\hline Scan Area & $\begin{array}{c}\text { Airborne } \\
\text { laser dataset }\end{array}$ & $\begin{array}{c}\text { Terrestrial laser } \\
\text { dataset }\end{array}$ \\
\hline Point cloud size & $9 \mathrm{MB}$ & $53 \mathrm{MB}$ \\
\hline $\boldsymbol{\sigma}$ (expected noise level) & $80 \mathrm{~cm}$ & $4 \mathrm{~cm}$ \\
\hline $\begin{array}{l}\text { K (number of neighboring } \\
\text { points for the local point } \\
\text { density calculation) }\end{array}$ & 15 & 50 \\
\hline $\begin{array}{l}\mathbf{n} \text { (reliable number of the } \\
\text { points for plane definition) }\end{array}$ & 12 & 15 \\
\hline Distance threshold $(\boldsymbol{\Delta d})$ & $50 \mathrm{~cm}$ & $10 \mathrm{~cm}$ \\
\hline Angle threshold $(\boldsymbol{\Delta} \boldsymbol{\alpha})$ & $\pi / 16 \mathrm{rad}$ & $\pi / 16 \mathrm{rad}$ \\
\hline $\begin{array}{l}\text { Size of the minimum } \\
\text { detectable cluster }\end{array}$ & 10 & 50 \\
\hline
\end{tabular}

Table1. Basic attributes and defined segmentation thresholds of provided datasets

The reliability and robustness of the newly developed technique will be examined through the following experiments.

\subsection{Airborne laser dataset}

Figure 7.a shows a region of the provided airborne laser dataset which has been selected for detailed investigation. This dataset includes gable roof shapes, terrain surface and vegetation 
(Figure7.b). Figure 7.c displays the results of grouping planar surfaces within the airborne laser data. The roof surfaces and the terrain surface are correctly grouped as planar surfaces.

Figure 7.d represents the output of the developed segmentation algorithm for this region based on the thresholds defined in Table 1. Each color in this figure represents a single planar surface.

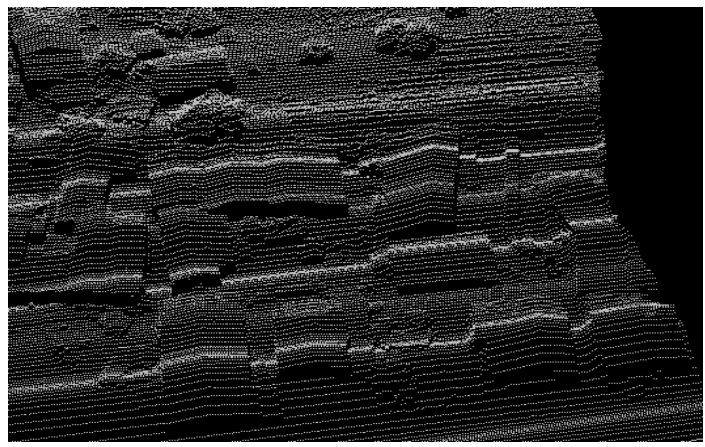

(a)

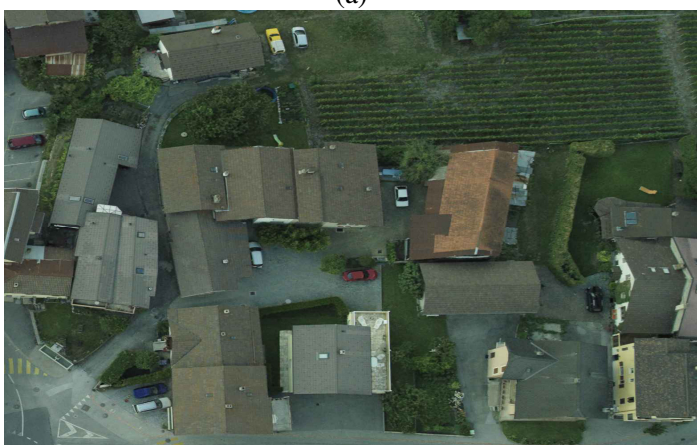

(b)

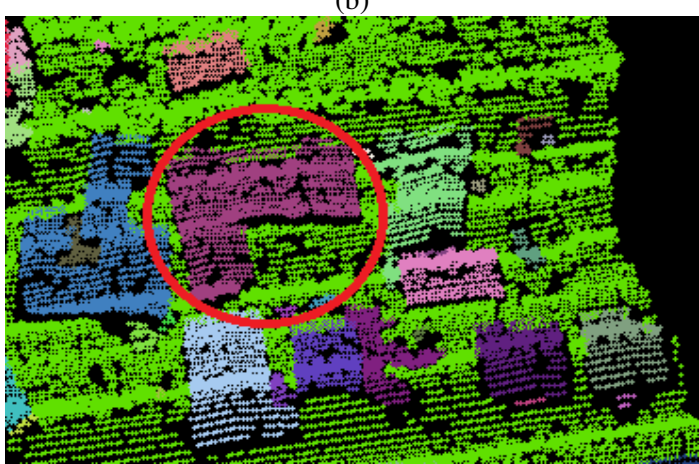

(c)

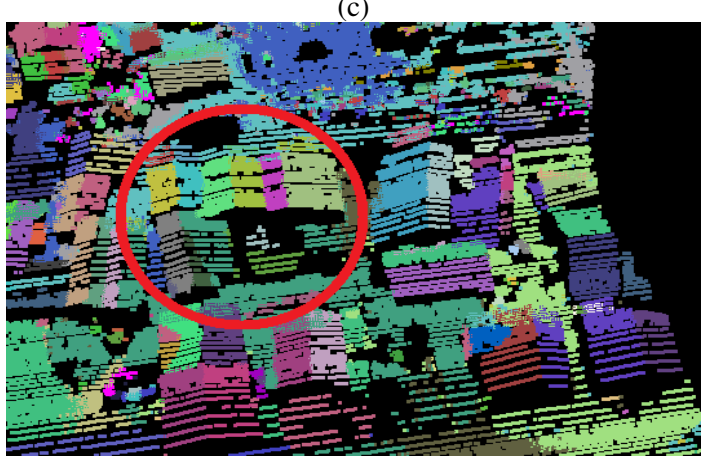

(d)

Figure 7. Airborne laser data, original point cloud (a), imagery (b), point classification result (c) and output of developed segmentation approach $(\mathrm{d})$
Comparison of represented segmentation results with grouping results (Figure 7.c) and image data (Figure 7.b) shows the feasibility of this methodology for segmentation of gable roofs (a sample is shown in red circles in Figures 7.c and 7.d), planar roofs with different slopes and terrain surface (Figure 7.d).

\subsection{Terrestrial laser data}

Figure 8.a shows a terrestrial laser scan of a building façade. Since this dataset contains planes with different slopes and much more variations in point density, it is more challenging than the airborne laser data.

Figure 8.c represents the results of grouping planar surfaces within the terrestrial laser data. The roof surfaces, walls and terrain surface are correctly grouped as planar surfaces.

Figure 8.d represents the output of the proposed segmentation approach for this laser point cloud based on the thresholds defined in Table 1. Each color in this figure represents a single planar surface.

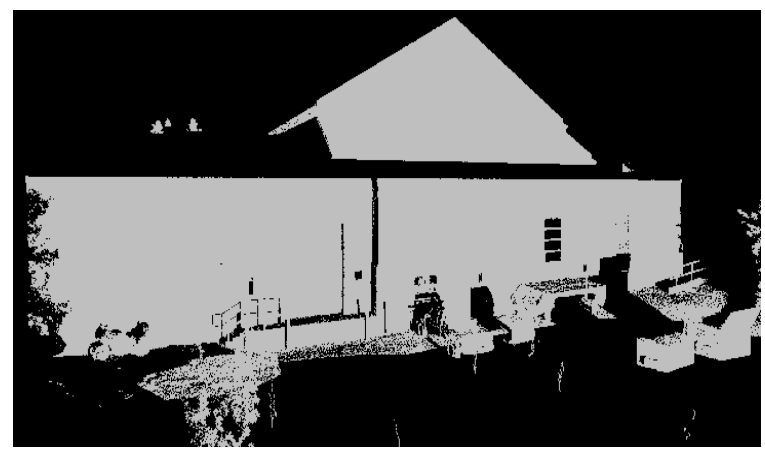

(a)

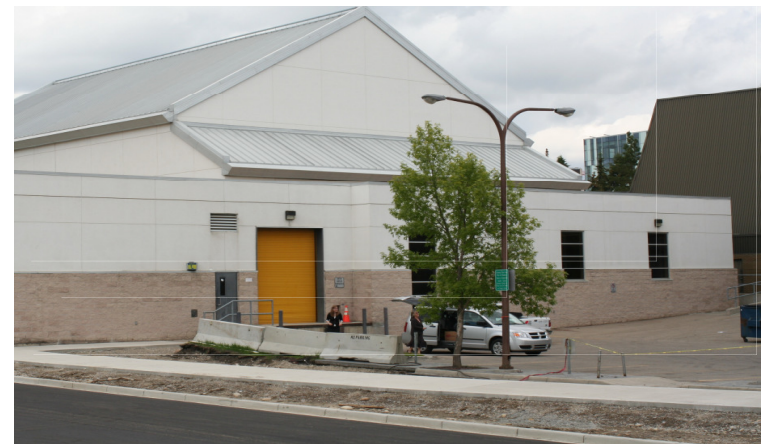

(b)

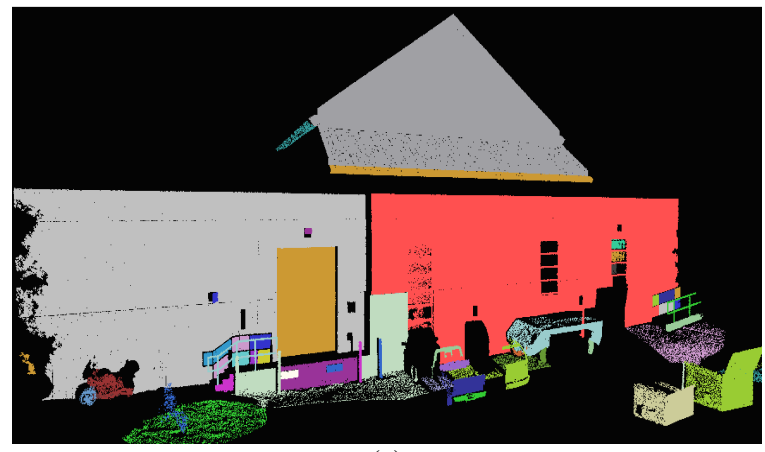

(c) 


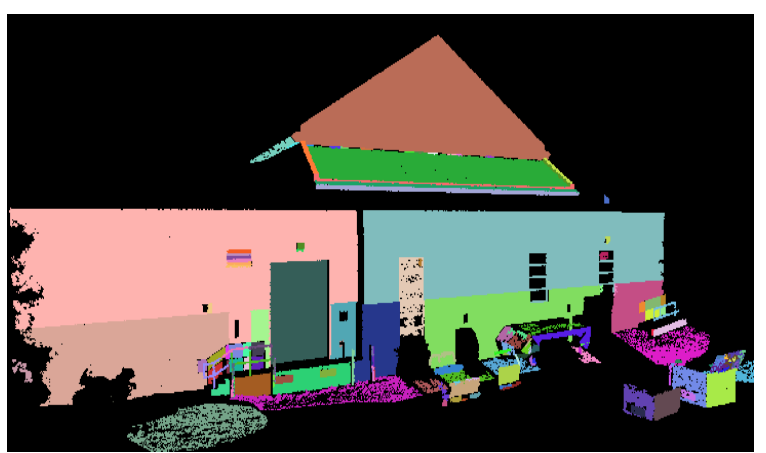

(d)

Figure 8. Terrestrial laser data, original point cloud (a) imagery

(b), point classification result (c) and output of developed segmentation approach $(\mathrm{d})$

Comparison of derived segmentation results with grouping results (Figure 8.c) and image data (Figure 8.b) proves the robustness of this methodology for the segmentation of parallel planes and intersecting planes with different slopes such as roof's planar surfaces with different slopes (Figure 8.d).

\section{CONCLUSIONS AND FUTURE WORK}

Extraction of spatial information from randomly distributed laser point cloud requires a reliable segmentation approach. Although many techniques have been developed for the segmentation of this kind of data, there are not many methodologies which can handle different laser point cloud datasets. This paper introduces a new technique for the segmentation of laser data based on clustering attributes which can be utilized for both airborne and terrestrial laser datasets with varying point densities and different noise levels.

In order to provide efficient access to the points and speed up the neighborhood definition process for the computation of segmentation attributes, a kd-tree structure is employed for the organization of the 3D laser data in this research. The adaptive cylinder method is then applied for neighborhood definition. This neighborhood system considers the local point density, expected noise level in the data and the local surface trend to increase the flexibility of proposed methodology. So, it can be utilized for point cloud from different sources. Afterwards, the coordinates of origin's normal projection on each point's best fitting plane are computed as the segmentation attributes. Finally, an octree-based space partitioning procedure is used for detection and extraction of the clusters in the attribute space. The main advantage of this clustering approach is its computational efficiency while considering the full attributes of the involved points.

Future research work will focus on quantitative evaluation of the proposed method segmentation results in comparison with the results derived from other well-known segmentation techniques. Later on, we will implement spatial analysis of the segmentation results for 3D feature extraction from both airborne and terrestrial laser data.

\section{ACKNOWLEDGEMENTS}

The authors would like to thank the GEOIDE (GEOmatics for Informed DEcisions) Network of Centers of Excellence (project: PIV_SII72) and Natural Sciences and Engineering Research Council of Canada Discovery and Strategic Project Grants for financial support of this research. They are also grateful to Dr. Jan Skaloud for providing the airborne laser dataset.

\section{REFERENCES}

Besl, P. J. and Jain, R. C., 1988. Segmentation through variable-order surface fitting. IEEE Transactions on Pattern Analysis and Machine Intelligence, 10(2), pp. 167-192.

Biosca, J. and Lerma, J., 2008. Unsupervised robust planar segmentation of terrestrial laser scanner point clouds based on fuzzy clustering methods. ISPRS Journal of Photogrammetry and Remote Sensing, 63(1), pp. 84-98.

Filin, S., 2002. Surface clustering from airborne laser scanning data. International Archives of Photogrammetry, Remote Sensing and Spatial Information Sciences, 34 (Part 3A), pp.117-124.

Filin, S., Pfeifer, N., 2005. Neighborhood systems for airborne laser data. Photogrammetric Engineering \& Remote Sensing, 71 (6), pp. 743-755.

Filin, S. and Pfeifer, N., 2006. Segmentation of airborne laser scanning data using a slope adaptive neighbourhood. ISPRS Journal of Photogrammetry and Remote Sensing, 60(2), pp. 7180.

Fischler, M. A. And Bolles, R., 1981. Random sample consensus: a paradigm for model fitting with applications to image analysis and automated cartography. Communications of the ACM, 24(6), pp. 381-395.

Friedman, J. H., Bentley, J. L. and Finkel, R. A., 1977. An Algorithm for Finding Best Matches in Logarithmic Expected Time. ACM Trans on Mathematical Software, 3(3), pp. 209226.

Hough, P.V.C., 1962. . US Patent 3066954, Methods and means for recognizing complex patterns.

Kim, C., Habib, A. and Mrstik, P., 2007. New approach for planar patch segmentation using airborne and terrestrial laser data". In: Proc. Of ASPRS annual conference, Tampa, Florida, USA.

Lee, I. and Schenk, T., 2001. Autonomous extraction of planar surfaces from airborne laser scanning data, In Proc. ASPRS annual conference, St. Louise, MO, USA.

Moore, A. W., 1991. An introductory tutorial on kd-trees. Report No. 209, Computer Laboratory, University of Cambridge, UK.

$\mathrm{Pu}$, S. and Vosselman, G., 2006. Automatic extraction of building features from terrestrial laser scanning. International Archives of the Photogrammetry, Remote Sensing and Spatial Information Sciences, 36(5), pp. 33-39.

Rabbani, T., Van den Heuvel, F. A. and Vosselman, G., 2006. Segmentation of point clouds using smoothness constraint. International Archives of Photogrammetry, Remote Sensing and Spatial Information Sciences, 36(5), pp. 248-253.

Tarsha-Kurdi, F., Landes, T. and Grussenmeyer, P., 2007. Hough-transform and extended RANSAC algorithms for automatic detection of $3 \mathrm{~d}$ building roof planes from lidar data, International Archives of Photogrammetry, Remote Sensing and Spatial Information Sciences, 36(3/W52), pp. 407-412.

Tovari, D. and Pfeifer, N., 2005. Segmentation based robust interpolation-a new approach to laser data filtering, International Archives of Photogrammetry, Remote Sensing and Spatial Information Sciences, 36(3/W19), pp. 79-84.

Vosselman, G., Dijkman, S., 2001. 3D building model reconstruction from point clouds and ground plans, International Archives of Photogrammetry and Remote Sensing, 34( 3/W4), pp. 37-43. 